\title{
Technetium Attenuation in the Vadose Zone: Role of Mineral Interactions
}

(Project Number: 70177)

\section{Principal Investigator}

Nancy J. Hess

Pacific Northwest National Laboratory

P.O. Box 999, MSIN P7-50

Richland, WA 99352

509-376-9808 (phone)

509-372-1632 (fax)

nancy.hess@pnl.gov

\section{Co-Investigators}

Steven D. Conradson

Los Alamos National Laboratory

MST-11, MS D429

Los Alamos, NM 87545

505-667-9584 (phone)

conradson@lanl.gov

James P. McKinley

Pacific Northwest National Laboratory

P.O. Box 999, MSIN K6-81

Richland, WA 99352

509-375-6841 (phone)

james.mckinley@pnl.gov

Kenneth M. Krupka

Pacific Northwest National Laboratory

P.O. Box 999, MSIN K6-81

Richland, WA 99352

509-376-4412 (phone)

ken.krupka@pnl.gov
Dhanpat Rai

Pacific Northwest National Laboratory P.O. Box 999, MSIN P7-50

Richland, WA 99352

509-373-5988 (phone)

dhan.rai@pnl.gov

Raymond E. Wildung

Pacific Northwest National Laboratory

P.O. Box 999, MSIN P7-54

Richland, WA 99352

509-376-5680 (phone)

r.wildung@pnl.gov 


\section{Research Objective}

High-level waste (HLW) has leaked into the vadose zone from buried single-shell tanks at the Hanford Site. Contaminant plumes containing radionuclides are slowly migrating toward the groundwater table. The accepted model of contaminant migration places technetium (Tc) at the leading edge of the plume due to the high mobility of the anionic species, $\mathrm{TcO}_{4}{ }^{-}$, in its oxidized state. However, recent drilling and sampling programs at the B-BX-BY and S-SX Hanford Site tank farms have revealed that the Tc distribution within the contaminant plume is more complex than anticipated and some Tc may be retained in the vadose zone as insoluble precipitates. This research project investigates possible mechanisms by which Tc associated with HLW leakages can be attenuated in the vadose zone. It also will provide fundamental information on surfacemediated reduction/precipitation reactions of $\mathrm{Tc}$ on $\mathrm{Fe}^{\mathrm{II}}$-containing mineral surfaces and the stability of Tc precipitates under conditions in the vadose zone at the Hanford Site. This information will provide much needed data for the development of models of Tc mobility in the vadose zone following closure of the tank farms. With reliable models, a major cost reduction in remediation efforts may be achieved by selecting sites that present the greatest environmental threat and designing remediation methods with the greatest efficacy.

\section{Research Progress and Implications}

This report summarizes work after 2.5 years of a 3-year study. We hypothesized that if Tc was to be retained in the vadose zone it must be present as a reduced precipitate. To test our hypothesis, we undertook three main tasks: 1) to evaluate the reductive capacity of Hanford formation sediments; 2) to determination of Tc speciation in contaminated Hanford formation sediments; and 3) to conduct solubility experiments under reducing conditions at which $\mathrm{Tc}^{\mathrm{IV}}$ species are stable in solution.

\section{Reductive Capacity}

The reductive capacity of Hanford formation sediments has been evaluated by two methods: using sequential chemical extractions to determine the distribution of $\mathrm{Fe}^{\mathrm{II}}$ and $\mathrm{Fe}^{\mathrm{III}}$ species on sediments and using one-dimensional flow experiments to test the capacity of Hanford sediments to reduce pertechnetate. Using the methods of Heron et al. (1994), untreated sediments were compared to sediments that had been pretreated with $4 \mathrm{M} \mathrm{NaOH}$ for $200 \mathrm{hr}$ and $450 \mathrm{hr}$.

Although little change was observed between untreated and $200 \mathrm{hr}$ pretreated sediments, the total quantity of $\mathrm{Fe}^{\mathrm{II}}$ and $\mathrm{Fe}^{\mathrm{III}}$ species more than doubled from $1260 \mu \mathrm{g} / \mathrm{g}$ to $2720 \mu \mathrm{g} / \mathrm{g}$ between the $200 \mathrm{hr}$ and $450 \mathrm{hr}$ pretreatments. The increase was largely due to an increase in availability of $\mathrm{Fe}^{\mathrm{III}}$ species. The number of $\mathrm{Fe}^{\mathrm{II}}$ surface sites was largely independent of pretreatment whereas

the number of ion-exchangeable Fe ${ }^{\mathrm{II}}$ species actually decreased upon pretreatment probably due to the precipitation of $\mathrm{Fe}(\mathrm{OH})_{2}$. 
The flow experiments were designed to simulate the geochemical conditions created by the leakage of HLW into the vadose zone and to test the reactions between pertechnetate and the sediments under caustic conditions. Solutions of oxygen-free $4 \mathrm{M} \mathrm{NaOH}$ and known concentrations of pertechnetate were injected into the loaded column and the concentration of $\mathrm{Tc}$ in the effluent was measured as a function of time. These experiments demonstrated that 1) the amount of Tc retained is independent of the concentration of Tc injected, 2) the lag in Tc retention observed between 1 and 2 pore volumes likely results from the gradual increase in $\mathrm{Fe}^{\mathrm{II}}$ concentration as Fe bearing mineral phases dissolve in the presence of $4 \mathrm{M} \mathrm{NaOH}$, and 3) the rate of Tc retention was also found to be weakly dependent on the flow rate through the column. Twelve sediment samples were removed from along the length of the column and subjected to various "oxidative conditions" to determine how tightly Tc was retained on the sediments. These conditions included oxygen-saturated water, $5 \mathrm{M} \mathrm{HCl}, 0.1 \mathrm{M} \mathrm{HNO}_{3}$, and $1 \mathrm{M} \mathrm{HNO}_{3}$. Oxygen-saturated water, $0.1 \mathrm{M} \mathrm{HNO}_{3}$ and $5 \mathrm{M} \mathrm{HCl}$ were relatively ineffective at oxidizing and remobilizing the Tc. Only $1 \mathrm{M} \mathrm{HNO}_{3}$ removed all the retained Tc. The failure of mild oxidative treatments to remove more than $25 \%$ of the Tc from the column sediments strongly suggests that Tc is present as reduced species that are relatively insoluble and resistant to re-oxidation.

Autoradiography and scanning electron microscopy (SEM) analysis of the caustically treated sediments were conducted to determine whether reduced Tc precipitate was preferentially associated with specific mineral phases, such as Fe-oxides, or whether it was homogeneously distributed over all mineral surfaces. Figure 1 shows an autoradiographic map of the six sediment samples from the column. The dark spots on the autoradiographic map indicate that the Tc is highly localized and associated with mineral grains. The arrow near Sample 10 points to a region that was selected for SEM analysis. Figure 2a is the SEM image of this area, and it shows two grains several hundred microns in diameter. The bright areas in the backscattered electron (BSE) image of this same region, Figure $2 b$, indicate concentrations of elements with large number of electrons, such as Fe. Figures 2c and $2 \mathrm{~d}$ are higher resolution BSE images of the grain on the right in Figures $2 \mathrm{a}$ and $2 \mathrm{~b}$. The white square in Figure 3d shows the area where an energy dispersive

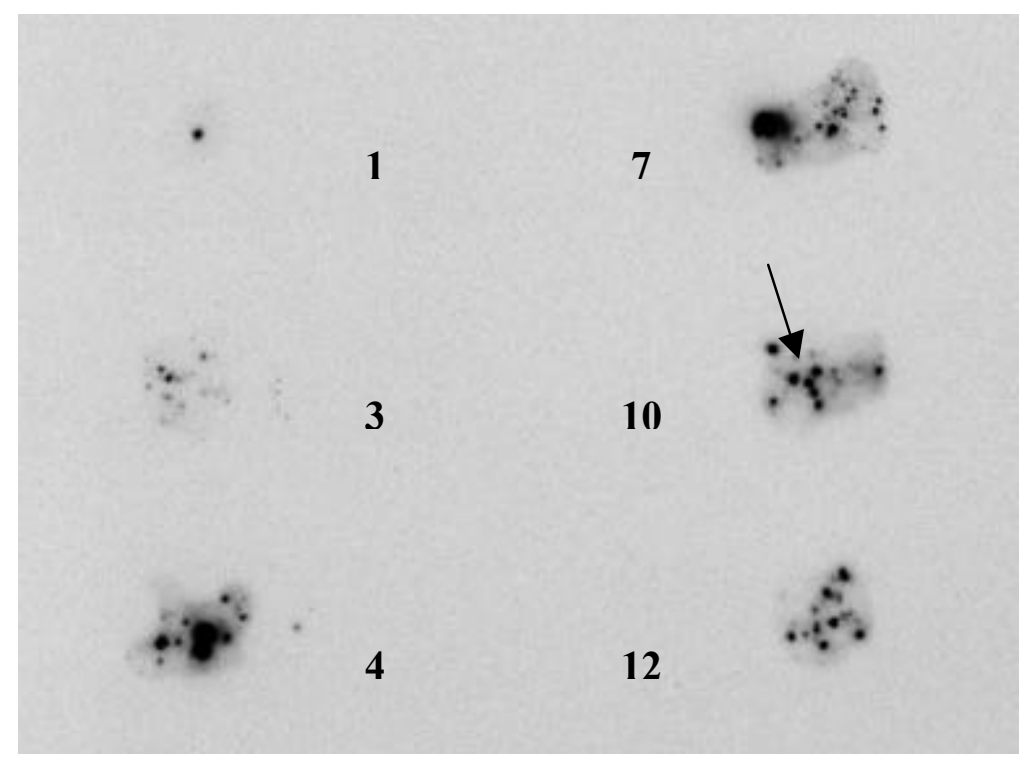

Figure 1. Autoradiographic map of six samples removed from the 1-D column. An arrow shows the location of the SEM studies in Figure 2. 

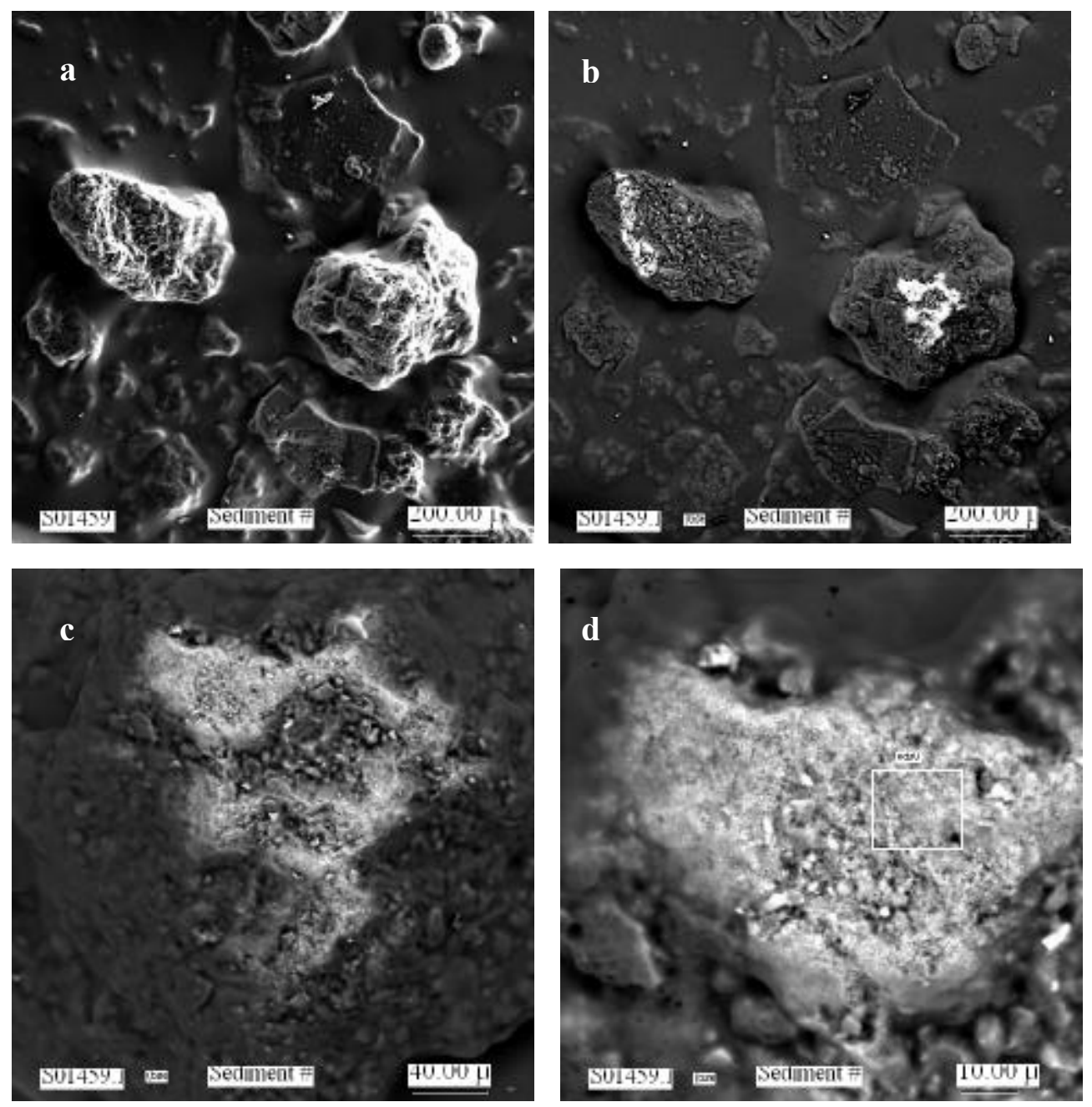

Figure 2. Scanning electron microscope images of Sample 10, Area 3 (indicated by arrow in Figure. 1). Figure $2 b$ is the backscattered electron (BSE) image of the region shown in Figure $2 a$. Figure $2 \mathrm{c}$ is a high-resolution BSE image of the grain on the left. Figure $2 \mathrm{~d}$, at higher resolution, shows the area where an energy dispersive spectrum, displayed in Figure 3, was aacquired.

spectrum (EDS) was acquired. The EDS, which gives the elemental composition of the scanned region, is shown in Figure 3. The presence of the elements $\mathrm{Si}, \mathrm{Al}, \mathrm{O}, \mathrm{Ca}, \mathrm{Na}$, and $\mathrm{K}$ reflect the abundant quartz and feldspar minerals that constitute the Hanford formation sediments. The presence of $\mathrm{Fe}$ and $\mathrm{Ti}$ in this spectrum indicates the presence of minor amounts of Fe-oxides, perhaps Ti-rich magnetite grains. Note that Tc could not be observed in the EDS spectrum because the Tc K emission is at too high an energy, and the Tc L emission intensity is too weak to be detected at the Tc concentrations present in the sample. However, there is a direct correlation between the dark spots on the autoradiographic map, which indicate the presence of Tc, and high concentrations of Fe in the EDS of the SEM images. 


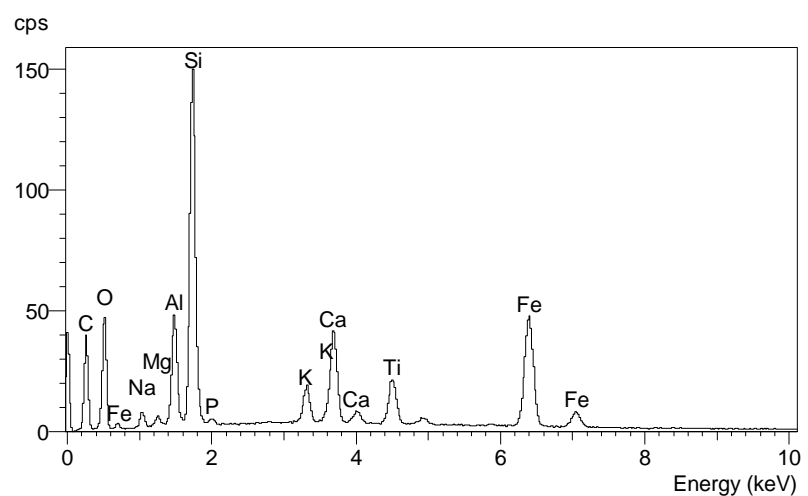

Figure 3. Energy dispersive spectrum of region indicated by the white box in Fig 7d. The intensity of Fe Ká and Kâ peaks indicate the presence of Fe-rich mineral phases.
The experimental results obtained under this task were critical in directing the research focus of the renewal proposal. We have demonstrated that the reductive capacity of Hanford sediments increases under basic conditions. In addition, we have found a set of experimental conditions that are relevant to the leakages of HLW from waste tanks at the Hanford Site under which pertechnetate is reduced by Hanford formation sediments. Furthermore, we observed evidence of the association of $\mathrm{Tc}$ with $\mathrm{Fe}$-oxides that suggests surface-mediated reduction of pertechnetate is the operable mechanism.

\section{Speciation of Hanford Formation Sediments}

To determine the speciation of the Tc in the vadose zone, and especially whether Tc is associated with Fe-oxides, Tc-contaminated samples were obtained from three boreholes drilled in the SX tank farm in the 200 West Area at the Hanford Site. The horizons were selected for either autoradiography or x-ray absorption spectroscopy (XAS) analysis. Samples from five horizons from the 299-W23-19 borehole (B8809) adjacent to Tank SX-115 were selected for autoradiography experiments because the radioactivity was exclusively due to ${ }^{99} \mathrm{Tc}$, and therefore, there would be no interference from other radionuclides such as ${ }^{137} \mathrm{Cs}$. Only one horizon, which consisted of a very fine sand, showed evidence of nonuniform Tc distribution, suggesting an association of Tc with a particular mineral phase. Unfortunately, efforts to isolate and identify the composition of these sediment grains using scanning electron microscopy were unsuccessful due to the small size of the soil particles.

Since the oxidation state of Tc is the dominant factor in predicting the mobility of Tc in the vadose zone, samples with high Tc concentrations were selected from two boreholes adjacent to tank SX-108 for Tc K-edge XAS studies on beamline 11-2 at the Stanford Synchrotron Radiation Laboratory. Through analysis of the x-ray absorption near edge structure (XANES) portion of the XAS spectrum, the oxidation state of Tc in the samples can readily be determined. The selected samples contained significant Tc contamination in both the water and acid extracts suggesting that Tc was predominantly present as pertechnetate. The samples were analyzed both "as-received" and "water-washed." Ideally, the as-received samples should contain Tc as pertechnetate whereas the water-washed treatment would remove the highly soluble pertechnetate, leaving any insoluble reduced $\mathrm{Tc}^{\mathrm{IV}}$ species behind. Unfortunately, even sample 4A/B/C, the horizon with the highest Tc contamination, contained insufficient Tc for bulk XAS 
analysis. Therefore, a fraction of this sample and sample 15A from the slant borehole were prepared for micro x-ray fluorescence mapping on the PNC-CAT beamline at the Advanced Photon Source.

The micro x-ray fluorescence technique uses an x-ray beam a few microns in diameter that is rastered over the surface area of the sample. The energy of the x-ray beam is sufficient to excite $\mathrm{x}$-ray fluorescence from elements of interest in the sample. The fluorescence spectrum is collected as a function of $x-y$ position providing an elemental map of the sample. Comparison of the Tc distribution to other elements may reveal associations between Tc and other mineral phases; for example, Fe-oxides. The results of micro x-ray fluorescence mapping for samples $15 \mathrm{~A}$ and $4 \mathrm{~A} / \mathrm{B} / \mathrm{C}$ are shown in Figure 4. The elemental maps for Fe, Mn, and Ti are strongly correlated in both samples whereas the elemental maps for $\mathrm{Fe}$ and $\mathrm{Ca}$ are inversely correlated.
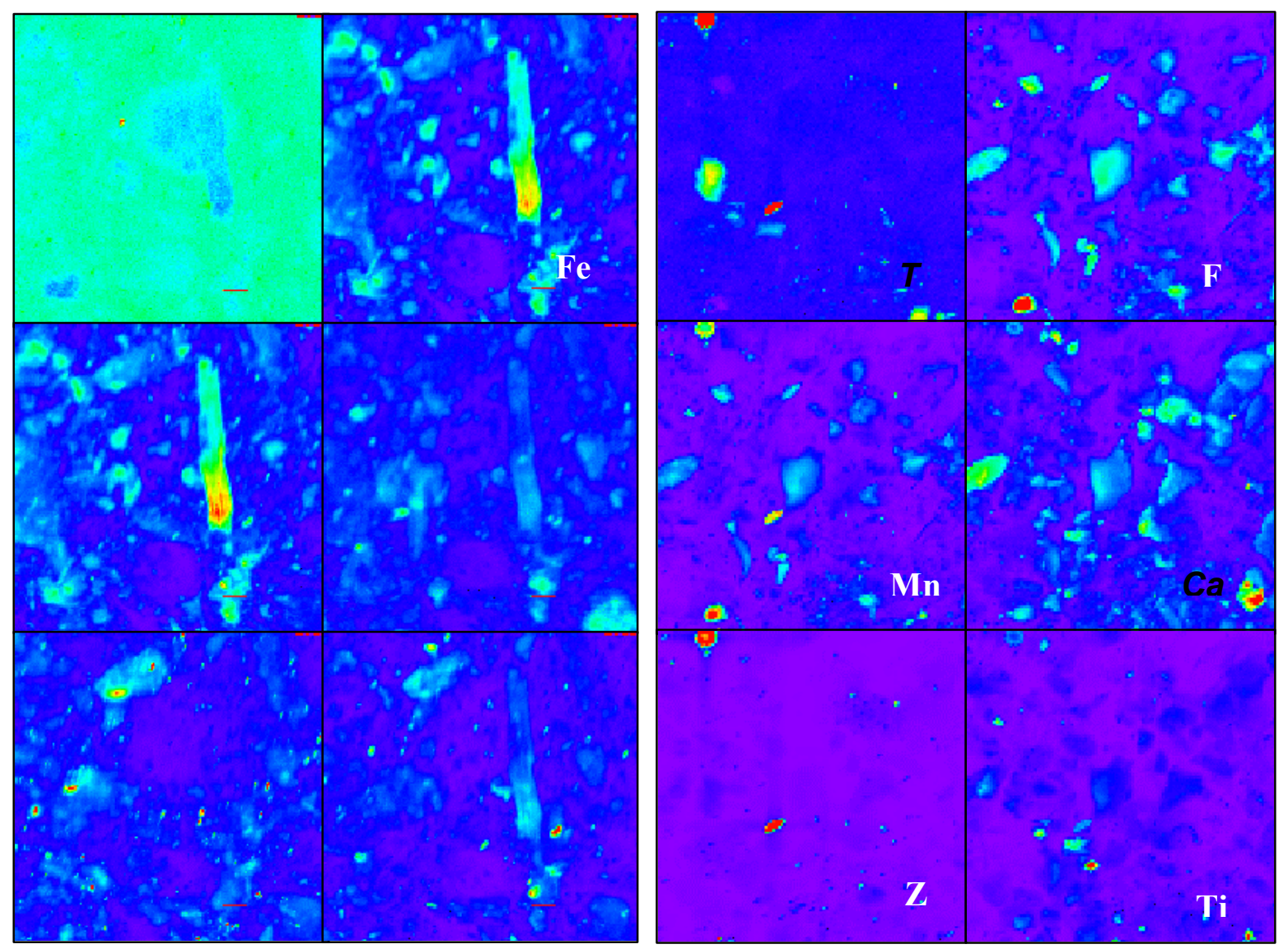

Figure 4. X-ray microprobe elemental maps for samples $15 \mathrm{~A}$, left, and $4 \mathrm{~A} / \mathrm{B} / \mathrm{C}$, right. Warm tones indicate high concentrations of the element indicated. The apparent Tc concentrations in sample $4 \mathrm{~A} / \mathrm{B} / \mathrm{C}$ are actually due to interference from $\mathrm{Zr}$. 
The Fe-Mn-Ti correlation is likely due to the presence of titanomagnetite and Fe-Mn oxide grains. The inverse correlation with Ca probably reflects the presence of feldspars and calcite grains. A detailed analysis of the Tc "hot spots" in sample 4A/B/C using micro-XANES indicated that the apparent Tc signal was due to interference from $\mathrm{Zr}$; the Tc K-edge at $21044 \mathrm{eV}$ and the $\mathrm{Zr} \mathrm{L}_{\mathrm{III}}$-edge at $22230 \mathrm{eV}$ are too close in energy to be completely resolved.

Efforts to characterize the Tc in contaminated Hanford formation sediments from the tank farm drilling program have been unsuccessful although the reported Tc concentrations should have been sufficient for the experimental techniques we are using. We are currently reviewing the sampling protocols at the drill site and our sample preparation methods to determine if either, or both, of these processes are removing Tc from the samples before analysis. We are hopeful that the drill cores from the B-BX tank farm will provide sediment samples that will permit direct determination of the Tc speciation.

\section{Solubility of Reduced Tc Species}

The solubility of reduced Tc species was determined under a wide range of $\mathrm{pH}$ conditions and in the presence of high concentrations of chloride and bicarbonate/carbonate. We conducted solubility studies of $\mathrm{TcO}_{2}(\mathrm{am})$ as a function of $\mathrm{pH}$ from $6 \mathrm{M} \mathrm{HCl}$ to $\mathrm{pH} 5$ and as function of chloride concentration from 0.001 to $5 \mathrm{M} \mathrm{NaCl}$. In addition, we have conducted these experiments under carefully controlled reducing conditions such that the preponderance of Tc is present in solution in the reduced $\mathrm{Tc}^{\mathrm{IV}}$ oxidation state. The oxidation state determination based on solvent extraction techniques has been verified by analysis of the XANES portion of the x-ray absorption spectra and UV-Vis spectroscopy, where the solution concentrations are high enough. Powder x-ray diffraction and analysis of the EXAFS portion of the x-ray absorption spectra were used to characterize the solid phase in equilibrium with the solution phase.

Our solubility measurements are in good agreement with those reported in the literature in the $\mathrm{pH}$ range from 1 to 5 as shown in Figure 5. At acidities less than $\mathrm{pH}=1$, there is increasing discrepancy. At a given $\mathrm{pH}$, increased solubility is observed with increased chloride concentration. Models of Tc solubility based on the existing thermodynamic data are in good agreement at low chloride concentrations and at $\mathrm{pH}$ greater than 1 but fail to predict the observed Tc solubility at high chloride concentration. EXAFS analysis of the solution species indicates that $\mathrm{TcCl}_{6}$ species dominate at $\mathrm{pH}$ less than or equal to zero. Unfortunately, the concentration of Tc species is not sufficient for EXAFS analysis at higher $\mathrm{pH}$, where mixed hydroxychloride Tc complexes and Tc hydrolysis products are thought to exist. Characterization of the solid phase by EXAFS analysis across the $\mathrm{pH}$ range indicates the stability of $\mathrm{TcO}_{2}(\mathrm{am})$ as the solubility 


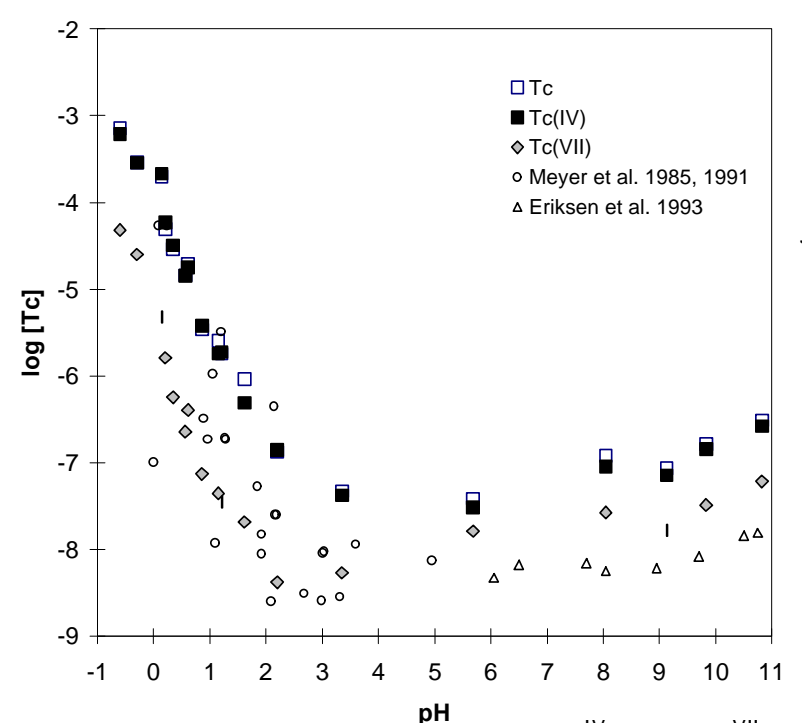

Figure 5a. Measured total Tc, $\mathrm{Tc}^{\mathrm{IV}}$, and $\mathrm{Tc}^{\mathrm{v}}$ in solution in contact with $\mathrm{TcO}_{2}(\mathrm{am})$. The reported Tc ${ }^{\text {IV }}$ data of Meyer and Eriksen are shown for comparison.

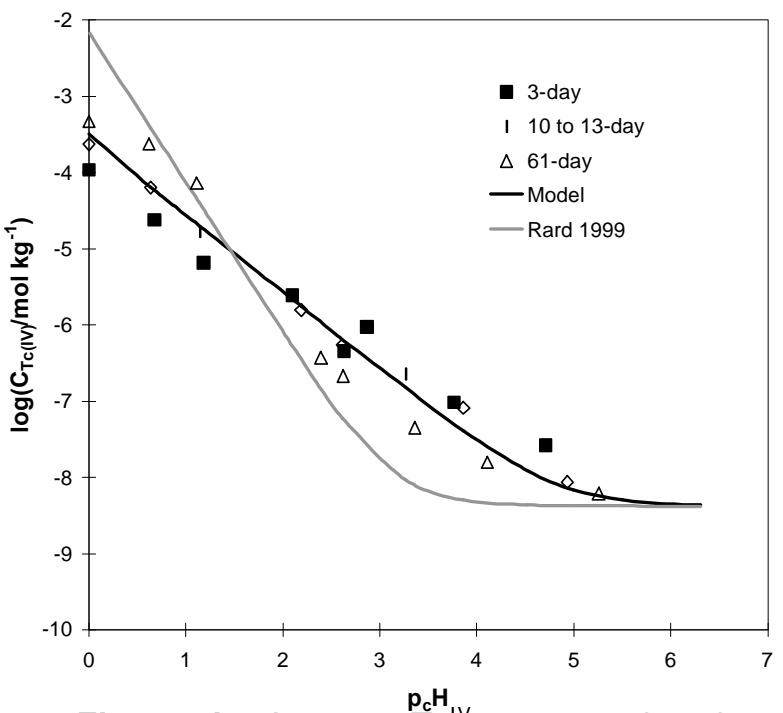

Figure 5b. Aqueous $\mathrm{Tc}^{\mathrm{p}_{\mathrm{H}} \mathrm{H}^{\mathrm{v}}}$ concentrations in contact with $\mathrm{TcO}_{2}(\mathrm{am})$ in $2.5 \mathrm{M} \mathrm{NaCl}$ solution. The dashed line represents the predicted Tc concentration based on the existing thermodynamic data (Rard 1999). The solid line represents our improved model.

limiting phase. Analysis of the powder x-ray diffraction data from the solid sample indicates that solid phase is amorphous across the $\mathrm{pH}$ range. Solubility studies under alkaline conditions and in the presence of carbonate have just been initiated.

\section{Planned Activities}

Future experimental work includes batch experiments of Tc(VII) sorption/reduction on hematite, magnetite, biotite, and hornblende; micro-XANES analysis of sediments from column experiments; and solubility experiments in the presence of carbonate at constant ionic strength. Future data analyses include thermodynamic modeling of the solubility data and interpretation of the column studies, which then will be incorporated into journal publications.

\section{Information Access}

\section{Journal Articles}

Hess NJ, Y Xia, DA Moore, and D Rai. 2002. Thermodynamic model for the solubility of $\mathrm{TcO}_{2} \cdot n \mathrm{H}_{2} \mathrm{O}$ in acidic solutions and high $\mathrm{NaCl}$ concentrations. To be submitted to Journal of Solution Chemistry. 
Szecsody JE, JP McKinley, and NJ Hess. 2002. The effect of hyperalkaline high level waste simulants on the retention of Tc in Hanford Formation sediments. To be submitted to Environmental Science and Technology.

\section{Presentations}

Hess NJ, K Krupka, J McKinley, D Rai, R Wildung, and S Conradson. 1999. Technetium Attenuation in the Vadose Zone: Role of Mineral Interactions, EMSP Kickoff Workshop, November 1999, Richland, Washington.

Hess NJ, Y Xia, D Rai, K Krupka, J McKinley, R Wildung, and S Conradson. 2000. Technetium Attenuation in the Vadose Zone: Role of Mineral Interactions, National DOE/EMSP Workshop, April 2000, Atlanta, Georgia.

Hess NJ, D Rai, J McKinley, K Krupka, R Wildung, and S Conradson. 2000. Technetium Attenuation in the Vadose Zone: Role of Mineral Interactions, FY2001 Vadose Zone Principal Investigator Workshop, November 2000, Richland, Washington.

Hess NJ, J Szecsody, J McKinley, D Rai, K Krupka, R Wildung, and S Conradson. 2001. Technetium Attenuation in the Vadose Zone: Role of Mineral Interactions, FY2002 Vadose Zone Principal Investigator Workshop, November 2001, Richland, Washington.

\section{Web addresses}

1999-2000 Progress Report. http://www.osti.gov/em52/2000projsum/70177.pdf

Poster Board EMSP Workshop, April 2000.

http://www.osti.gov/em52/NWS2000_Posters/id70177.pdf 